\title{
Use of Artificial Intelligence/Machine Learning in Cancer Research During the COVID-19 Pandemic
}

\author{
Vineet Kumar Kamal ${ }^{1}$, Dolly Kumari²
}

${ }^{1}$ Scientist C, Division of Epidemiology and Biostatistics, ICMR-National Institute of Epidemiology, Chennai, India. ${ }^{2}$ Assistant Professor, Centre for Economic Policy and Public Finance, Asian Development Research Institute, Patna, India.

\begin{abstract}
The cancer patients are more vulnerable and are at increased risk of COVID-19 and related outcomes due to their weakened immune systems, specially patients with lung cancer. Amid pandemic, the diagnosis, treatment, and care of cancer patients are very difficult and challenging due to several factors. In such situations, the latest technology in artificial intelligence (AI) or machine learning algorithms (ML) have potential to provide better diagnosis, treatments and cares of cancer patients. For example, the researches may use clinical and imaging data with machine learning techniques to make differences between coronavirus-related lung changes and those caused by immunotherapy and radiotherapy. During this pandemic, AI can be used to ensure we are getting the right patients enrolled speedily and more efficiently than the traditional, and complex ways in the past in cancer clinical trials. This is the appropriate time to go beyond the "research as usual" approach and update our research via $\mathrm{AI}$ and $\mathrm{ML}$ tools to care the cancer patients and discover new and more effective treatments.
\end{abstract}

Keywords: Artificial intelligence- cancer care- corona virus- COVID-19- machine learning

\section{Introduction}

The novel coronavirus, also known as SARS-Cov-2 or COVID-19 has created a global health crisis and has become the biggest threat as a serious viral infection worldwide in very short span of time. Though COVID-19 can affect people of all ages, the cancer patients are more vulnerable and are at increased risk of developing the virus due to their weakened immune systems. According to a recently published study based on Chinese cohort, patients with cancer had a higher risk of evolving severe events like intensive care unit (ICU) admission, invasive ventilation, or death as compared to patients without cancer (39\% vs $8 \%$ ) [1]. In cancer care, this observation controlled many oncologists to alter their daily practice, however, cancer patients need continuous care, specially, in case of lung cancer, which continues to be the main cause of cancer-related deaths in men and women globally [2]. The Corona virus is known to affect and harshly impact the respiratory system, therefore, patients with lung cancer are largely vulnerable to this virus.
Amid pandemic, in cancer patients, undergoing diagnostic tests or therapeutic interventions is not a very easy job, in fact, it is very challenging, whereas their potential COVID-19 exposure could be hazardous, or even fatal. Also, clinical presentation of COVID-19 and diagnosing this disease in patients with cancer is challenging due to several factors. Cancer patients might have unusual radiographic features [3], or such patients might have radiographic findings similar to those of a SAR-CoV-2 infection and can be confusing and misleading [4]. The diagnosis of COVID-19 may be delayed due to similarity in symptoms between the infection and the underlying disease, particularly in lung cancer patients and patients with pulmonary metastasis. Also, some clinical and biological features can hide a COVID-19 presentation in cancer patients [5].

In all these situations, the latest technology in artificial intelligence (AI) or machine learning (ML) algorithms have potential to provide better care and treatments for

Corresponding Author:

Dr. Vineet Kumar Kamal

Scientist C, Division of Epidemiology \& Biostatistics, ICMR-National Institute of Epidemiology, Chennai, India.

Email: vineetstats@gmail.com 
cancer patients during the COVID-19 pandemic. AI and ML algorithms are transforming our lifestyle intending to mimic human intelligence by a computer/machine using statistical and mathematical models in solving various issues. AI is more sophisticated at doing what humans do, but more efficiently, rapidly, and at a lower cost in solving complex problems. AI has potential to provide an upper hand undeniably in clinical decision-making, in fact, it is providing. ML, a subset of AI, can find patterns from huge complex datasets to become more precise and accurate as they interact with training data, allowing humans to increase unprecedented insights into early finding of diseases, drug discovery, diagnostics, healthcare processes, treatment variability, and patient outcomes [6]. AI/ML algorithms have potential to fight against COVID-19 in many ways, for example, to identify who is most at risk using multivariable data from clinical or population settings, to diagnose patients, to identify natural interaction between predictors, or to find risk cluster, to develop drugs faster, in finding existing drugs that can help, to predict the spread of the disease, to understand viruses better, to map where viruses come from, and to predict the next pandemic [6-7-8-9].

During the pandemic clinicians have confronted a challenge of identifying whether lung changes in cancer patients are due to COVID-19, or if they are being caused by another infection or side effects from cancer treatment. Immunotherapy and radiotherapy are used to treat patients in some cancers. However, in many cases, these treatments' side effects like cough, breathlessness, and changes in CT scans can often be very similar to those seen in COVID-19 infection. To address this issue, a new study, led by The Royal Marsden NHS Foundation Trust in collaboration with The Institute for Cancer Research (ICR) and Imperial College London, has been started using AI [10]. In this study, the primary aim is to use AI to identify to what extent changes seen on chest scans are due to coronavirus or the side effects of cancer treatment. Secondary aim is to use AI to diagnose the subtle changes seen in lung cancer recurrence earlier so that it might be treated more effectively. The researches will use clinical and imaging data with ML techniques to help distinguish between coronavirus-related lung changes and those caused by immunotherapy and radiotherapy. Distinguishing between the two is very important in terms of treatment options: for example, cancer therapy-related lung inflammation is frequently treated with steroids and stronger immunosuppression, which are not appropriate for use in COVID-19 patients.

Many clinical trials and in-lab research for cancer care and treatments are hampered badly due to this pandemic, but some clinical trials cannot be put on hold and if we do not do so, the primary endpoint will be affected for sure and it would be very hard to ensure optimum outcome in trial settings, and, in some cases, deviating from a trial's planned protocol will take place. In normal days, participation rates in cancer treatment trials are already low, but the Covid-19 threatens to drive them even lesser. It has been established that that using an artificial intelligence algorithm can dramatically increase trial screening efficiency of oncologists and enable participation of small practices, which are frequently left out from trial enrollment and it can improve the patient identification timeline by nearly 90 percent [11]. So, AI virtually eradicates human disruption and potential misses caused by pandemics or other factors. The time has come to embrace AI to ensure we are getting the right patients enrolled speedily and more efficiently than the traditional, complex and inefficient ways of the past.

In summary, COVID-19 is compelling the entire health care ecosystem to work together to classify creative solutions that go beyond the "research as usual" approach. In case of cancer care and treatments, and for prognosis and diagnosis of cancer patients, during this pandemic and post pandemic, we need to look beyond old protocols and start to embrace a future that can maximize outcomes for patients. Updating our research via artificial intelligence and machine learning tools have potential to discover new and more effective cancer treatments that improve patient outcomes and save lives.

\section{References}

1. Liang W, Guan W, Chen R, Wang W, Li J, Xu K, Li C, Ai Q, Lu W, Liang H, Li S, He J. Cancer patients in SARSCoV-2 infection: a nationwide analysis in China. The Lancet Oncology. 2020 03;21(3):335-337. https://doi.org/10.1016/ s1470-2045(20)30096-6

2. Fitzmaurice C, Dicker D, Pain A, Hamavid H, Moradi-Lakeh M, MacIntyre MF, Allen C, et al. The Global Burden of Cancer 2013. JAMA Oncology. 201507 01;1(4):505. https:// doi.org/10.1001/jamaoncol.2015.0735

3. Qu J, Yang R, Song L, Kamel I. Atypical lung feature on chest $\mathrm{CT}$ in a lung adenocarcinoma cancer patient infected with COVID-19. Annals of Oncology. 2020 06;31(6):825-826. https://doi.org/10.1016/j.annonc.2020.03.001

4. Zhu W, Wang J, He X, et al. [The differential diagnosis of pulmonary infiltrates in cancer patients during the outbreak of the 2019 novel coronavirus disease]. Zhonghua Zhong Liu Za Zhi. 2020;42(4):305-11. https://doi.org/10.3760/ cma.j.cn112152-20200303-00166

5. Jin X, Zheng KI, Pan K, Xie Y, Zheng M. COVID-19 in a patient with chronic lymphocytic leukaemia. The Lancet Haematology. 2020 04;7(4):e351-e352. https://doi. org/10.1016/s2352-3026(20)30074-0

6. Dananjayan S, Raj GM. Artificial Intelligence during a pandemic: The COVID -19 example. The International Journal of Health Planning and Management. 202005 20;35(5):1260-1262. https://doi.org/10.1002/hpm.2987

7. Trebeschi S, Drago S, Birkbak N, Kurilova I, Călin A, Delli Pizzi A, Lalezari F, Lambregts D, Rohaan M, Parmar C, Rozeman E, Hartemink K, Swanton C, Haanen JBAG, Blank C, Smit E, Beets-Tan R, Aerts H. Predicting response to cancer immunotherapy using noninvasive radiomic biomarkers. Annals of Oncology. 2019 06;30(6):998-1004. https://doi.org/10.1093/annonc/mdz108

8. Jiang X, Coffee M, Bari A, Wang J, Jiang X, Huang J, Shi J, Dai J, Cai J, Zhang T, Wu Z, He G, Huang Y. Towards an Artificial Intelligence Framework for Data-Driven Prediction of Coronavirus Clinical Severity. Computers, Materials \& Continua. 2020;62(3):537-551. https://doi.org/10.32604/ cmc.2020.010691

9. Kamal VK, Srivastav S, Kumari D, Ranjan M. Identification of distinct risk subsets for under five mortality in India 
using CART model: an evidence from NFHS-4. Journal of Global Health Reports. 202006 17;. https://doi. org/10.29392/001c.13169

10. Using AI technology to benefit cancer patients during the COVID-19 pandemic | The Royal Marsden NHS Foundation Trust. Accessed July 25, 2020. https://www.royalmarsden. nhs.uk/using-ai-technology-benefit-cancer-patients-duringcovid-19-pandemic.

11. Ni Y, Wright J, Perentesis J, Lingren T, Deleger L, Kaiser M, Kohane I, Solti I. Increasing the efficiency of trial-patient matching: automated clinical trial eligibility Pre-screening for pediatric oncology patients. BMC Medical Informatics and Decision Making. 201504 14;15(1). https://doi. org/10.1186/s12911-015-0149-3

(c) (1) (9)

This work is licensed under a Creative Commons AttributionNon Commercial 4.0 International License. 